\title{
Mass Selective Ejection by Axial Resonant Excitation from a Linear Ion Trap
}

\author{
Yuichiro Hashimoto, Hideki Hasegawa, Takashi Baba, and Izumi Waki \\ Central Research Laboratory, Hitachi, Ld., Tokyo, Japan
}

We describe a new mass selective ejection method from a linear ion trap, which we call axial resonant excitation (AREX). A set of vane lenses are inserted between each quadrupole rod to produce electrostatic potential that is approximately harmonic along the central axis of the quadrupole field. After ions with specific $\mathrm{m} / \mathrm{z}$ are resonantly oscillated in the axial direction, the ions are mass selectively ejected in the axial direction. At a high scan rate of $11 \mathrm{Th} / \mathrm{ms}$, AREX achieved a high ejection efficiency of more than $60 \%$, which is more than three times higher than a conventional mass selective axial ejection method from a linear trap using fringing field. (J Am Soc Mass Spectrom 2006, 17, 685-690) (C) 2006 American Society for Mass Spectrometry

$\mathrm{M}$ $\mathrm{S}^{n}(n \geq 3)$ analysis by a quadrupole ion trap is a powerful tool for analyzing proteins [1], peptides [2], carbohydrates [3], metabolites, and other compounds [4]. The quadrupole ion trap can be classified into two types, the 3D-quadrupole ion trap (QIT) or the 2D-quadrupole ion trap (or linear ion trap, LIT). The LITs are widely used as a mass selective ejection device in a low-resolution mass spectrometer $[5,6]$ or a fragmentation and precursor selection device in a higher-resolution hybrid mass spectrometer [7-9], because the LIT has $\sim 10$ times higher trapping efficiency and two times broader trapping "mass window" than the QIT [5, 9]. For a mass selective ejection device, the ejection efficiency and scan rate is very important because these determine the sensitivity and throughput of the mass spectral acquisition. A scan rate of 1-10 $\mathrm{Th} / \mathrm{ms}$ is used for commercial instruments, so as not to degrade the sensitivity and the throughput [5, 6]. Schwartz et al. reported mass selective ejection in a radial direction with an ejection efficiency of $44 \%$ at a scan rate of $5.5 \mathrm{Th} / \mathrm{ms}$ [5]. After ions are oscillated by a supplemental AC field applied between a pair of opposite quadrupole rods, they are ejected through the exit slits of the rods into dual detectors. Hager reported mass selective ejection in an axial direction with the ejection efficiency of less than $20 \%$ at a scan rate of 1.0 $\mathrm{Th} / \mathrm{ms}$ [6]. Though ions were radially oscillated in a similar manner as that of Schwartz, the fringing field near the end lens ejects the radially oscillated ions into the axial direction. Collisions of the ions with the rods decreased the ejection efficiency to less than $20 \%$. In a similar axial ejection, a better axial-ejection efficiency of over $90 \%$ was achieved by a high-order field perturba-

Published online March 9, 2006

Address reprint requests to Dr. Y. Hashimoto, Central Research Laboratory, Hitachi, Ltd., 1-280 Higashi-koigakubo, Kokubunji-shi, Tokyo 185-8601, Japan. E-mail: h-yuichi@crl.hitachi.co.jp tion with a low scan rate of $0.02 \mathrm{Th} / \mathrm{ms}$ [10]. This scan rate is too slow for practical use, because it degrades the duty cycle of the LIT to less than $1 \%$.

We report in this paper a new mass selective axial ejection with high ejection efficiency of $60 \%$ at 11 $\mathrm{Th} / \mathrm{ms}$ by axial resonant excitation (AREX) in an LIT. A set of lenses that are inserted between each quadrupole rod makes a DC potential that is approximately harmonic along the central $\mathrm{z}$-axis of the linear trap. Inside the quasi-harmonic potential, ions with a specific $\mathrm{m} / \mathrm{z}$ range can be resonantly oscillated in the axial direction by superimposing a supplemental AC field, so that the ions are mass-selectively ejected in the axial direction. High ejection efficiency is possible, because the oscillated and ejected directions are the same.

\section{Experimental}

\section{Instrumentation}

The experimental setup was modified from the linear trap mass spectrometer described previously [9] and is depicted in Figure 1. Samples are prepared as $100 \mathrm{nM}$ solutions in a methanol solution, and are ionized in a positive mode using a gas-assisted electrospray ion source (ESI), at a flow rate of $5 \mu \mathrm{l} / \mathrm{min}$. Ions are introduced from the ESI through a multipole ion guide into a pre-trap. The pre-trap can isolate ions with a specific $m / z$ as described [9]. Helium is introduced into the pre-trap as a bath gas of 2 mTorr. After trapping ions for about $50 \mathrm{~ms}$, the ions are ejected out of the pre-trap into an AREX LIT. The AREX LIT has an incap lens, an endcap lens, four quadrupole rods, and eight lenses that are inserted between the rods (vane lenses). The rod length of the AREX LIT is $44 \mathrm{~mm}$, the rod radius $(R)$ is $9.17 \mathrm{~mm}$, and the distance between the rod and the center axis $(r)$ is $8.00 \mathrm{~mm}$. Two phases of the trapping RF voltage ( $\pm 600 \mathrm{~V}(0-$ peak $), 770 \mathrm{kHz})$ were 


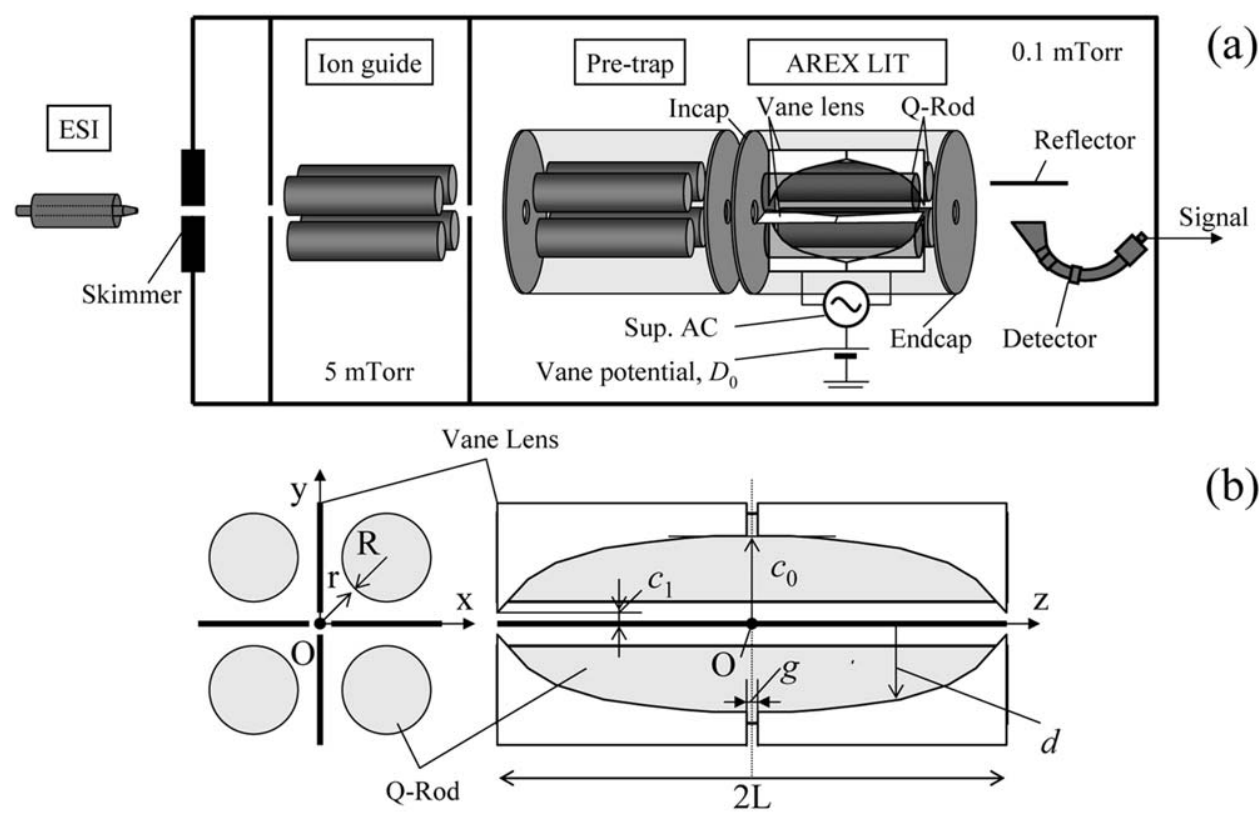

Figure 1. Schematic drawing of AREX LIT; $c_{0}: 11.6 \mathrm{~mm}, c_{1}: 0.8 \mathrm{~mm}, L: 22 \mathrm{~mm}, g: 1.0 \mathrm{~mm}$, and $p$ : fitting parameter $(1.0-3.0)$.

applied to the rod pairs. Vane lenses consist of a set of four front vanes and another set of four rear vanes, with a thickness of $0.5 \mathrm{~mm}$. The four lenses comprising each set are at a same electric potential. The vane potential $\left(D_{0}\right)$, which is a DC potential of the vane lenses relative to a quadrupole-rods DC offset, makes a potential that is approximately harmonic along the central axis of the quadrupole field. The shape of the vane lenses and the potential formed thereby are described in the next section. A supplemental AC voltage $(< \pm 20.0 \mathrm{~V}(0-$ peak), 5-100 kHz) was applied between the front and rear vanes to oscillate ions resonantly in an axial direction. The oscillated ions were ejected and detected in an axial direction.

The measurement sequence of the AREX LIT is shown in Figure 2. The measurement sequence consists of accumulation, cooling, scan, and emptying periods. During an accumulation period, the ions from the pre-trap are accumulated inside the AREX LIT, where the incap and the endcap lenses make an axial trapping field, and quadrupole rods make a radial trapping field inside the trap. Helium was introduced into the AREX LIT as a 2 mTorr bath gas, where the base pressure of the chamber was about $2 \times 10^{-5}$ torr. Typical trapping efficiency was over $90 \%$, which is consistent with the other LIT [9]. During a cooling period, the vane potential $\left(D_{0}\right)$ rises to $10-150 \mathrm{~V}$, so that ions are focused near a central point $O$. During a scan period, a supplemental $\mathrm{AC}$ frequency is swept from a higher to a lower frequency at a constant scan rate. Oscillated ions were mass-selectively ejected and detected by an electron multiplier (Burle Industries Inc., Lancaster, PA). During an emptying period, all the remaining ions in the AREX are ejected by lowering endcap DC potential.

\section{Geometry of Vane Lenses to Approximate Axial Harmonic Potential}

To create approximately a harmonic potential along the $z$-axis, we used eq 1 as a model of vane lens shape. The distance, $d$, between the edge of the vane lens and the central axis is

$$
d=c_{0}+\left(c_{1}-c_{0}\right)\left(\frac{|z|}{L}\right)^{p}
$$

where $c_{0}, c_{1}$, and $L$ describe physical dimensions of the vane lenses as in Figure 1b.

We calculated a best-fit potential whose difference from the harmonic is minimized within the z-range of $\pm 0.8 \mathrm{~L}$. The fitting equation is

$$
D(z)=D_{0}\left(A_{0}+A_{2}\left(\frac{z}{L}\right)^{2}\right),
$$

where $A_{0}$ and $A_{2}$ are free fitting parameters. Within this fitting range, the vane potential sufficiently approximates a harmonic potential, though it deviates from the harmonic near the edge of the rod. Figure 3 shows the correlation coefficient between the harmonic potential and the vane potential within $\pm 0.8 \mathrm{~L}$ for various $p$ values. When $p$ is from 1.5 to 2.2 , the correlation coefficient is above 0.998 , so that the potential approximates a harmonic potential sufficiently. Therefore, we chose a vane lens with $p=2.0$ in all later experiments. 


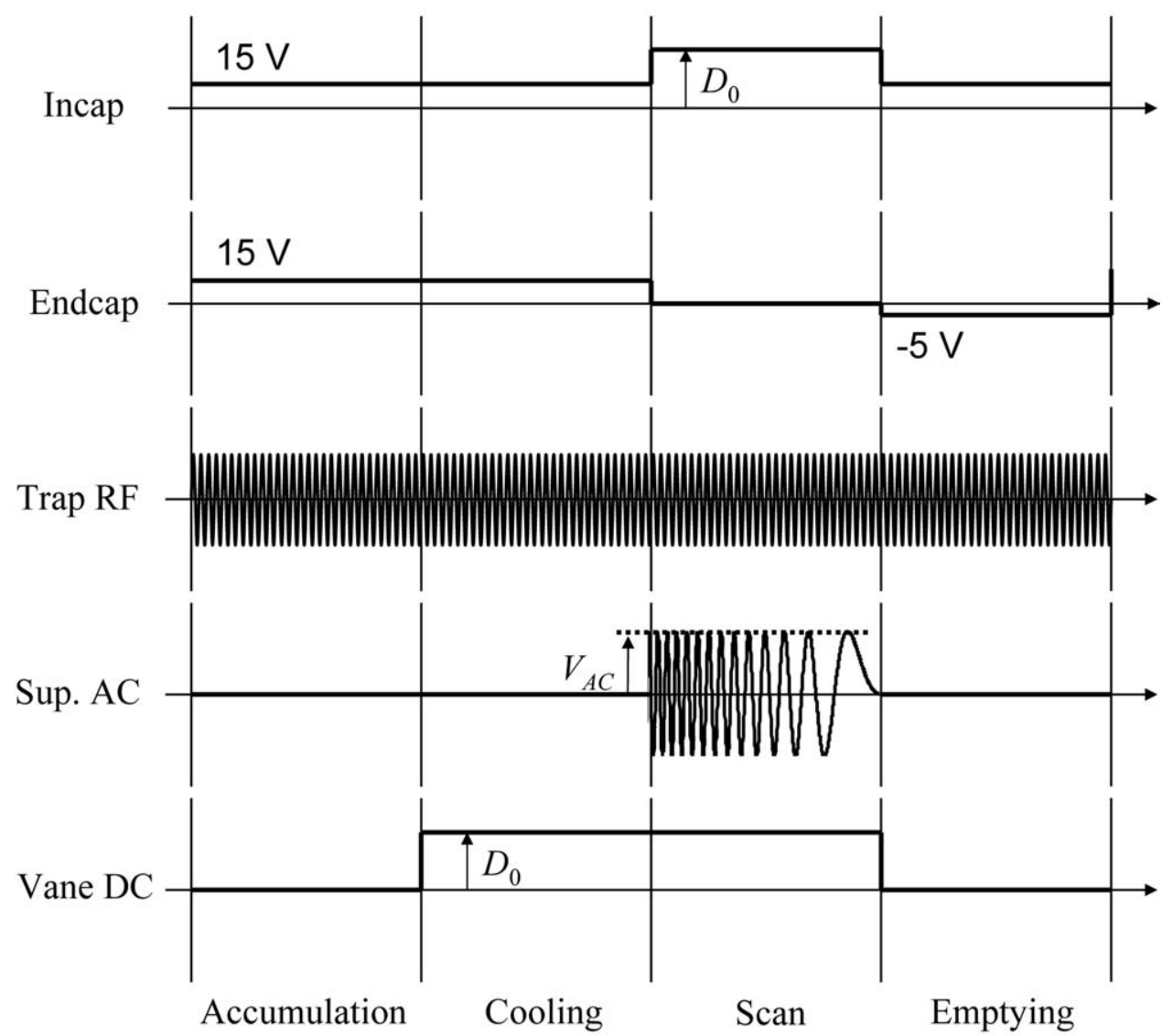

Figure 2. Measurement sequence of AREX LIT.

\section{Results and Discussion}

\section{Mass Resolution of AREX}

From eq 2, axial ion motion is given by

$$
m \frac{d^{2} z}{d t^{2}}=-e \frac{\partial}{\partial z} D(z)=-2 e A_{2} D_{0} \frac{z}{L^{2}},
$$

where $m$ is a mass of ions. The resonant frequency in the axial direction, $f$, is obtained from eq 3 .

$$
f=\frac{1}{2 \pi} \sqrt{\frac{2 e A_{2} D_{0}}{m L^{2}}} .
$$

Eq 4 can be differentiated to give

$$
\frac{m}{d m}=-\frac{1}{2} \frac{f}{d f}
$$

Therefore, the mass resolution by a frequency sweep was defined by $\frac{f_{0}}{2 \Delta f}$, where $f_{0}$ is a center frequency of the peak and $\Delta f$ is a full width at half maximum. Figure 4 shows a frequency sweep spectrum of reserpine peak at 609.3 Th. The fine line is the spectrum obtained by a single scan and the bold line is an averaged spectrum of 128 scans. Substituting the bestfit $A_{2}$ into eq 4 , we obtained a calculated resonant frequency of $42.33 \mathrm{kHz}$, which meets well with the observed $f_{0}$ range between 42.1 and $42.8 \mathrm{kHz}$ (Figure 4). Frequency was swept from higher to lower at various sweep rates between -50 and $-1600 \mathrm{~Hz} / \mathrm{ms}$, which corresponds to mass scan rates of $1.4 \mathrm{Th} / \mathrm{ms}$ and $46.3 \mathrm{Th} / \mathrm{ms}$ at $609.3 \mathrm{Th}$. Several subpeaks are observed in each single-scan spectrum. The

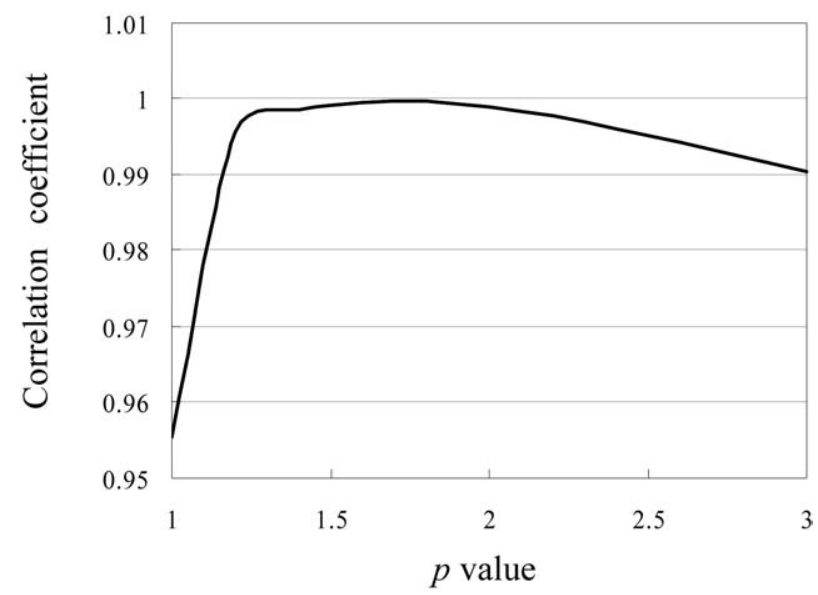

Figure 3. Correlation coefficient versus $P$ value. 

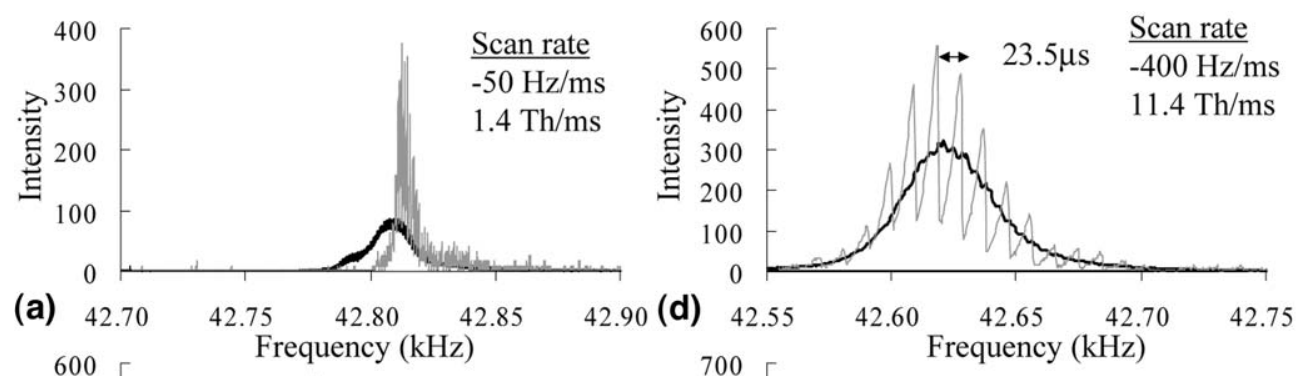

$\begin{array}{lrr}\text { (a) } 42.70 & 42.75 \quad 42.80 \quad 42 \\ 600 & \text { Frequency }(\mathrm{kHz})\end{array}$
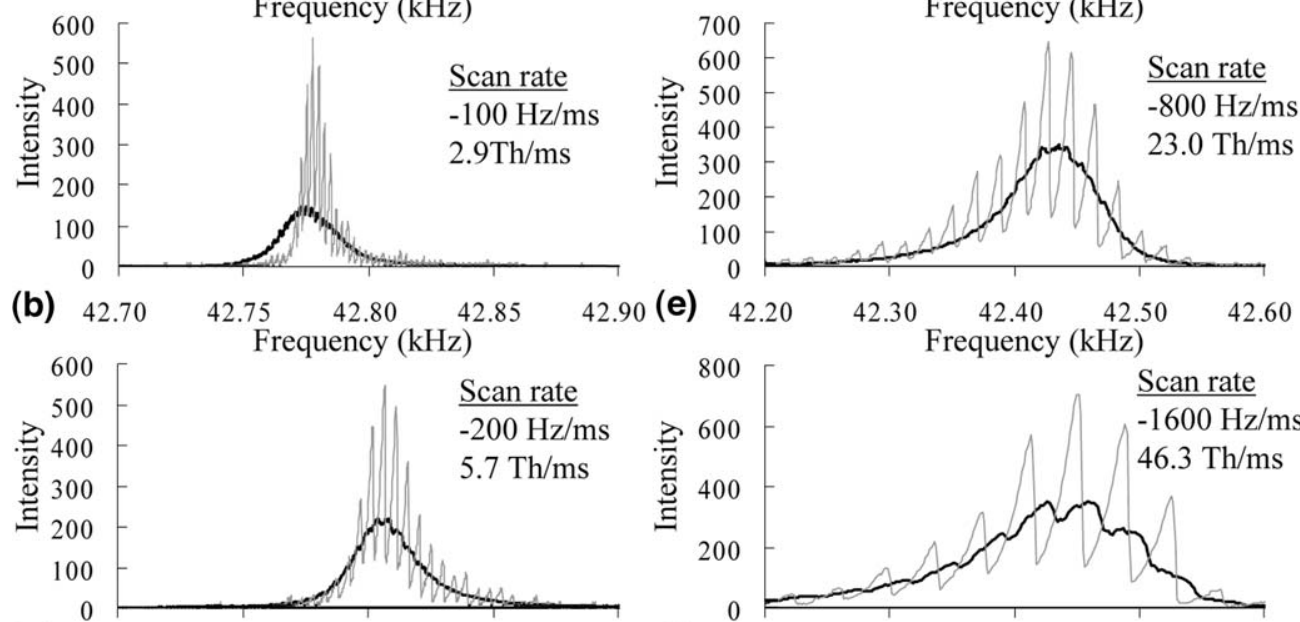

(c) 42.60

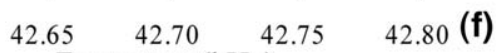
Frequency $(\mathrm{kHz})$

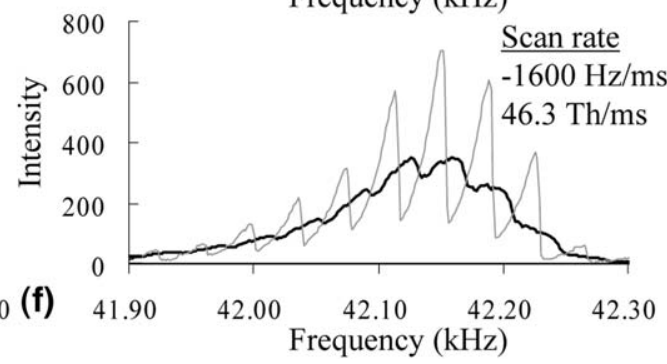

Figure 4. Frequency spectra for reserpine ion; bold line: average spectra of 128 scans, thin line: single scan spectra, $m / z=609.3, D_{0}=130 \mathrm{~V}$, supplemental AC amplitude was $12 \mathrm{~V}$.

time interval between sub-peaks is 23 to $24 \mu \mathrm{s}$, which corresponds to a resonance cycle of an axial harmonic potential. These sub-peaks appear because ions are ejected in a specific phase range of the supplemental AC field independently of main RF phase. In conventional LIT, the excited ions are also ejected synchronously with the supplemental AC. The sub-peaks are not observed, however, because signal detection response is too slow to resolve the sub-peaks, where the supplemental AC frequency is above several hundreds $\mathrm{kHz}$. On the other hand, the supplemental AC frequency of AREX was less than $50 \mathrm{kHz}$.

All the spectra by a single scan have a similar envelope shape with full width at half maximum containing 5 sub-peaks. The number of sub-peaks was similar in case of different scan rate and different vane potential $D_{0}$. Therefore, we suppose this envelope distribution derives from the thermal distribution of initial trapping state. The constant number of sub-peaks in a single scan envelope results in the frequency-width being inversely proportional to the scan rate, as can be seen in Figure $4 \mathrm{a}-\mathrm{f}$.

Mass resolution that is calculated from the envelope width was 1800 at a scan rate of $-50 \mathrm{~Hz} / \mathrm{ms}$ (Figure 4a). The resonance frequency of each single scan fluctuated by about $15 \mathrm{~Hz}$ because of the instability of the DC power supplies of about $100 \mathrm{mV}$, which is about $0.1 \%$ of the vane potential of $130 \mathrm{~V}$. Therefore, the mass resolution of the averaged spectrum in Figure 4a degraded to 900 .

Mass resolution calculated from the averaged peak was plotted in Figure 5. Though a lower mass scan rate achieves a higher mass resolution, the mass resolution

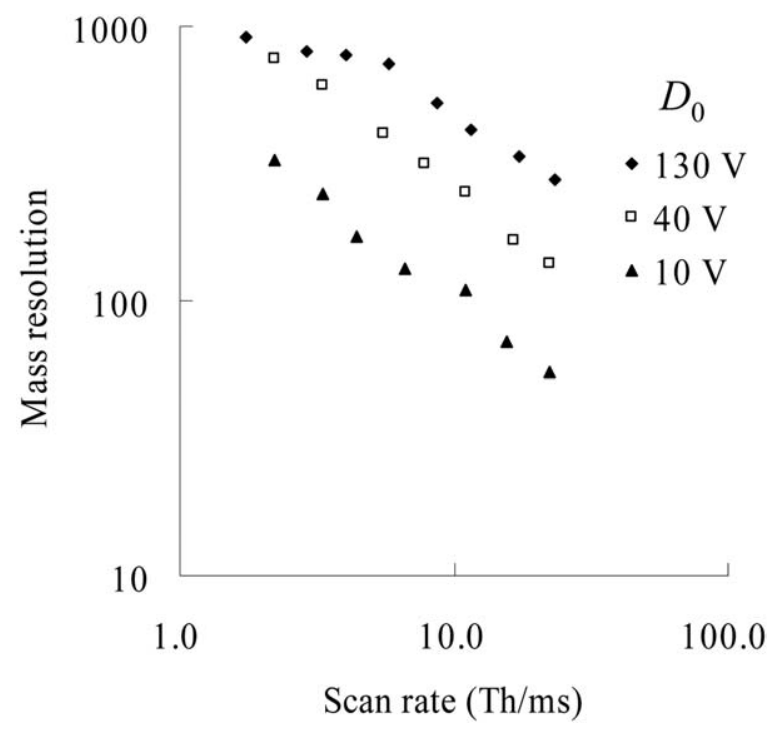

Figure 5. Mass resolution versus scan rate; $m / z=609.3$, supplemental AC amplitudes were 12, 6, $1.25 \mathrm{~V}$ for $D_{0}=130,40,10 \mathrm{~V}$, respectively. 
saturates below 1000 because of the instability of the electric supply as described. Above a scan rate of 6 $\mathrm{Th} / \mathrm{ms}$ with $D_{0}=130 \mathrm{~V}$, the mass resolution degrades inverse-proportionally to the scan rate, which is consistent with the fact that about five cycles $(5 / f)$ of harmonic oscillation corresponds to the averaged peak width as shown in Figure 4c-f. That is,

$$
\begin{aligned}
& \text { Mass_Scan_Rate }=\frac{d m}{d t}=\frac{\Delta m}{5 / f} . \\
& \text { Mass_Resolution }=\frac{m}{\Delta m}=m \frac{f / 5}{\text { Mass_Scan_Rate }} .
\end{aligned}
$$

In Figure 5, mass resolution is proportional to the root of $D_{0}$ at higher scan rates, which is consistent with the fact that about five cycles $(5 / f)$ of harmonic oscillation corresponds to the averaged peak width in different $D_{0}$ case. That is, mass resolution can be converted by eqs 4 and 7 to give

$$
\text { Mass_Resolution } \infty f \infty \sqrt{D_{0}} \text {. }
$$

\section{Ejection Efficiency of AREX}

Ejection efficiency was evaluated as follows. First, a pre-trap isolated reserpine ion of 609 Th from others so that all ions ejected from the AREX LIT are considered as reserpine ions. The initial amount of reserpine ions trapped in the AREX LIT was measured by axially ejecting all the trapped ions by lowering the endcap DC potential after ion accumulation. After resonantly ejecting the trapped ions, the remaining ions were measured by lowering the endcap DC of end lens. Figure 6 shows resonant ejection ions and remaining ions that are normalized by the initial amount of reserpine ions. High ejection efficiencies of more than $60 \%$ were obtained in a $V_{A C}$ range of $12-18 \mathrm{~V}$. Each mass resolution

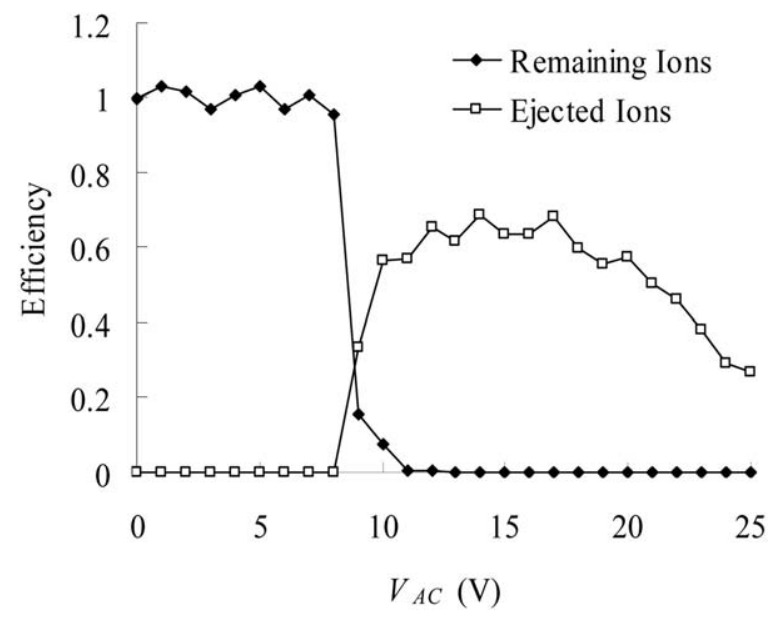

Figure 6. Ion ejection efficiency versus supplemental AC amplitude $V_{A C}$; scan speed: $-0.4 \mathrm{kHz} / \mathrm{ms} ; 11.4 \mathrm{Th} / \mathrm{ms}, D_{0}: 130 \mathrm{~V}$.

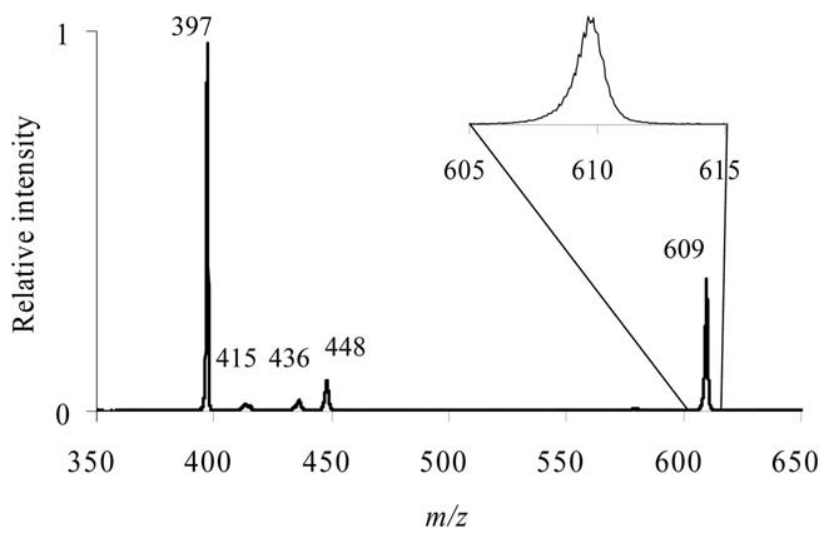

Figure 7. Mass Spectrum obtained by frequency sweep; scan speed: $-0.3 \mathrm{kHz} / \mathrm{ms}(57-42 \mathrm{kHz}) ; 8.6 \mathrm{Th} / \mathrm{ms}$ at $\mathrm{m} / \mathrm{z}=609.3$, scan period: $50 \mathrm{~ms} D_{0}: 130 \mathrm{~V}, V_{A C}: 15 \mathrm{~V}$.

ranged from 350 to 450 FWHM (typically Figure 4d). This high ejection efficiency is possible because the oscillated and ejected directions are the same, whereas the oscillated and ejected directions are perpendicular in the conventional axial ejection [6]. More than $50 \%$ of the ions are ejected into a detector direction, not into the pre-trap direction, because the incap lens potential (130 V) was higher than the endcap. When a same DC potential of $0 \mathrm{~V}$ was applied to the incap and endcap lens, ejection efficiency decreased to $30 \%$. The loss of ions is mainly estimated by the collisions with the endcap lens, which is also supported by simulation results. Figure 7 shows the mass spectrum that was converted from a frequency spectrum. In addition to the reserpine peak of 609 Th, fragment peaks of 397, 415, 436 , and $448 \mathrm{Th}$ are observed, which were produced at the skimmer. The mass resolution of each peak ranged from 350 to 500 .

As described above, AREX LIT achieved high trapping efficiency and high ejection efficiency, which are beneficial to practical use. On the other hand, AREX LIT might be affected by space charge more strongly than the other LITs because AREX LIT excites ions at a center point and the other LITs excite ions along the entire length of the quadrupole rods, which we will plan to evaluate in the future.

\section{Conclusions}

In summary, we demonstrated, for the first time, a mass selective ejection method from a linear ion trap, the axial resonant excitation (AREX). AREX achieved a high ejection efficiency of more than $60 \%$ at a mass resolution of 400 and a scan rate of $11 \mathrm{Th} / \mathrm{ms}$. We expect that the mass resolution of AREX can be improved further by optimizing the vane shape. This high efficiency of mass selective axial ejection at practical scan rate should also be beneficial for hybrid mass spectrometers. 


\section{Acknowledgments}

The authors acknowledge the many suggestions received concerning ion simulation by Dr. K. Yoshinari of Hitachi, Ltd., Hitachi Research Laboratory and Mr. H. Toyama and Mr. T. Iwata of the authors' laboratory for the electrical supply system provided for this instrument.

\section{References}

1. Coon, J. J.; Ueberheide, B.; Syka, J. E. P.; Dryhurst, D. D.; Ausio, J.; Shabanowitz, J.; Hunt, D. F. Protein identification using sequential ion/ion reactions and tandem mass spectrometry. Proc. Natl. Acad. Sci. U.S.A. 2005, 102, 9463-9468.

2. Kokubu, M.; Ishihama, Y.; Sato, T.; Nagasu, T.; Oda, Y. Specificity of immobilized metal affinity-based IMAC/C18 tip enrichment of phosphopeptides for protein phosphorylation analysis. Anal. Chem. 2005, 77(16), 5144-5154.

3. Ashline, D.; Singh, A.; Hanneman, A.; Reinhold, V. Congruent strategies for carbohydrate sequencing. 1 . Mining structural details by $\mathrm{MS}^{n}$. Anal. Chem. 2005, 77(19), 6250-6262.
4. Hopfgartner, G.; Husser, C.; Zell, M. Rapid Screening and characterization of drag metabolites using a new quadrupole-linear ion trap mass spectrometer. J. Mass Spectrom. 2003, 38(2), 138-150.

5. Schwartz, J. C.; Senko, M. W.; Syka, J. E. P. A two-dimensional quadrupole ion trap mass spectrometer. J. Am. Soc. Mass Spectrom. 2002, 13, 659-669.

6. Hager, J. W. A new linear ion trap mass spectrometer. Rapid Commun. Mass Spectrom. 2002, 16, 512-526.

7. Syka, J. E. P.; Marto, J. A.; Bai, D. L.; Horning, S.; Senko, M. W.; Schwartz, J. C.; Ueberheide, B.; Garcia, B.; Busby, S.; Muratore, T.; Shabanowitz, J.; Hunt, D. F. J. Proteome. Res. 2004, 3, 621-626.

8. Makarov, A.; Denisov, E., Lange, O.; Kholomeev, A.; Horning, S. Dynamic range of mass accuracy in FTMS. Proceedings of the 53rd ASMS Conference on Mass Spectrometry and Allied Topics; San Antonio, TX, June, 2005.

9. Hashimoto, Y.; Hasegawa, H.; Waki, I. Dual linear ion trap/orthogonal acceleration time-of-flight mass spectrometer with improved precursor ion selectivity. Rapid Commun. Mass Spectrom. 2005, 19, 1485-1491.

10. Moradian, A.; Douglas, D. J. Axial ion ejection from linear quadrupoles with added octopole fields. Proceedings of the 53rd ASMS Conference on Mass Spectrometry and Allied Topics; San Antonio, TX, June, 2005 